\title{
On stability of switched differential algebraic systems - conditions and applications *
}

\author{
Ishan Pendharkar* Kai Wulff** Jörg Raisch ${ }^{* * *}$ \\ * Max-Planck-Institute for Dynamics of Complex Technical Systems, \\ Magdeburg, Germany. \\ ** Control Systems Group, Technische Universität Berlin, Germany \\ (corresponding author, e-mail: wulff@control.tu-berlin.de) \\ *** Max-Planck-Institute for Dynamics of Complex Technical Systems, \\ and Control Systems Group, Technische Universität Berlin, Germany
}

\begin{abstract}
We obtain an efficient parametrization that ensures stability of switched linear systems under arbitrary switching. Apart from stability analysis, our results are useful for addressing several important system theoretic problems, e.g. designing controllers that ensure robustness against arbitrary combinations of sensor or actuator failure. We illustrate our results by considering control of a distillation column.
\end{abstract}

\section{INTRODUCTION}

In this paper we address the problem of stability analysis of switched linear systems described by high order differential algebraic equations. Switched linear systems are a special class of Hybrid systems. Hybrid systems have gained widespread importance in the recent years, since these provide a conceptually appealing framework for modelling of physical systems [13].

Switched linear systems, as the name suggests, are those in which every component system is linear. These systems have been extensively investigated, see e.g. $[2,4,16]$ for a survey. Systems that switch among a finite number of stable linear systems may still exhibit instability. Therefore it is of interest to characterize those stable linear systems that preserve stability under switching. We consider the case of arbitrary switching. This case is practically relevant when a switching sequence is a'priori unknown, or too complicated to enable a detailed specific analysis or simulation. Most results available on stability analysis of switched systems are of a numerical nature. The few theoretical results that are available are mostly sufficient conditions and only consider first order state space systems having a special structure. We show that there are practically important problems where numerical approaches fail, and therefore there exists a strong case for a deeper investigation of stability theory for switched systems. We develop a detailed theory for stability analysis which succeeds in relaxing some of the assumptions commonly made in this area. Our results are not only theoretically appealing but also practically relevant and useful: we use them to also address some important system theoretic problems related to stability under switching conditions.

This paper is organized as follows: In Section 2 we summarize the notation used throughout the paper. This is

\footnotetext{
* This work was partially supported by the European Union funded HYCON Network of Excellence, contract number FP6-IST-511368.
}

followed by a concise problem statement and literature overview in Section 3. In Section 4 we introduce a practical stability analysis problem for a trickling filter for which commonly available numerical algorithms fail. Section 5 introduces some of the tools and concepts necessary for the analytical treatment presented in this paper. Section 6 is the main section of the paper. We revisit the trickling filter in Section 7 and show how our results help us obtain an insight into the nature of the problem. Section 8 discusses further applications of our results - especially, designing controllers that ensure stability under arbitrary combinations of sensor / actuator failure where we also address a control problem for a distillation column. Due to limitations of space, we are forced to omit all proofs of our results. These however are included in [10], and available on request.

\section{NOTATION}

We denote the field of real numbers by $\mathbb{R}$, and that of complex numbers by $\mathbb{C}$. $\mathbb{R}^{\mathrm{m}}$ denotes the set of column vectors over $\mathbb{R}$ having $\mathrm{m}$ rows. $I_{\mathrm{m}}$ and $0_{\mathrm{m}}$ denote the $\mathrm{m} \times \mathrm{m}$ Identity, and Zero matrices, respectively. $\mathbb{R}^{\mathrm{q} \times \mathrm{m}}$ denotes the set of $\mathrm{q} \times \mathrm{m}$ matrices over $\mathbb{R} . \mathbb{R}^{\mathrm{q} \times \mathrm{m}}[D]$ denotes the set of $\mathrm{q} \times \mathrm{m}$ polynomial matrices over $\mathbb{R}$ in the indeterminate $D$ and $\mathbb{R}^{\mathrm{q} \times \mathrm{p}}[\zeta, \eta]$ denotes the set of $\mathrm{q} \times \mathrm{p}$ polynomial matrices in the indeterminates $\zeta$ and $\eta$. $\left.\mathbb{R}^{\mathrm{q} \times} \cdot \zeta, \eta\right]$ denotes the set of polynomial matrices in $\zeta, \eta$ having $\mathrm{q}$ rows and an unspecified number of columns. Given $Q \in \mathbb{R}^{\mathrm{q} \times \mathrm{m}}[D]:=$ $\sum_{i=0}^{d} \mathrm{Q}_{i} D^{i}, \mathrm{Q}_{i} \in \mathbb{R}^{\mathrm{q} \times \mathrm{m}}$, with $\mathrm{Q}_{d}$ a nonzero matrix, $d$ is called the degree of $Q$ and is denoted by $\operatorname{deg} Q$. Further, if $\mathbf{Q}_{d}$ is nonsingular, $Q$ is called a regular polynomial matrix. If $\mathrm{Q}_{d}=I, Q$ is called monic. If all roots of $\operatorname{det} Q=0$ lie in the open left half complex plane, $Q$ is called Hurwitz. Given two vector spaces $\mathcal{V}_{1}, \mathcal{V}_{2}$ and a linear operator $\mathcal{K}: \mathcal{V}_{1} \rightarrow \mathcal{V}_{2}$, $\operatorname{Ker} \mathcal{K}$ denotes the kernel of $\mathcal{K}$ while $\operatorname{Im} \mathcal{K}$ denotes the image of $\mathcal{K}$. 


\section{PROBLEM STATEMENT AND LITERATURE REVIEW}

Consider a linear MIMO system defined by a proper rational matrix $G=P Q^{-1}$, where $P$ and $Q$ are polynomial matrices, with $\operatorname{deg} Q-\operatorname{deg} P=d$. Consider a set $\mathcal{K}$, of polynomial differential operators of degree at most $d$, and define:

$$
\mathcal{Q}_{\mathcal{K}}:=\left\{Q_{K}=Q+K P \mid K \in \mathcal{K}\right\}
$$

The dynamical system

$$
\Sigma_{\mathcal{Q}_{\mathcal{K}}}:=Q(t)\left(\frac{d}{d t}\right) w=0, \quad Q(t) \in \mathcal{Q}_{\mathcal{K}}
$$

is called a switched linear system. $Q(t)$ can be thought of as a map from the set of non-negative real numbers $\mathbb{R}^{+}$to the space of polynomial matrices having degree $\operatorname{deg} Q$. The points of discontinuity of $Q(t)$ are called the "switching instances" and systems $Q_{K}\left(\frac{d}{d t}\right) w=0$ with $Q_{K} \in \mathcal{Q}_{\mathcal{K}}$ are called component systems of $\Sigma_{Q_{K}}$. Note that singularities of $Q+K P$ are precisely the closed loop poles of $G$ with a negative feedback $K$. Thus, the problem statement implicitly also includes a multivariable output feedback controller design problem, especially in cases when $K$ is assumed to be constant.

The central problem that we address in this paper is: "Obtain a parametrization for $\mathcal{K}$ such that the equilibrium state 0 in the switched system $\Sigma_{\mathcal{Q}_{\mathcal{K}}}$, remains asymptotically stable under arbitrary switching". With some abuse of notation we say that $\Sigma_{\mathcal{Q}_{\mathcal{K}}}$ is asymptotically stable if the equilibrium state 0 is asymptotically stable. Note that it is of course necessary for the stability of $\Sigma_{\mathcal{Q}_{\mathcal{K}}}$ that every component system is stable. Stability of every component system is however not sufficient to guarantee that the equilibrium in $\Sigma_{\mathcal{Q}_{\mathcal{K}}}$ is stable under arbitrary switching.

Notice that when every matrix $Q \in \mathcal{Q}_{\mathcal{K}}$ is a regular first order polynomial, $\Sigma_{\mathcal{Q}_{\mathcal{K}}}$ defines a switched state space system of the type:

$$
\Sigma_{\mathcal{A}}: \dot{x}(t)=A(t) x(t), A(t) \in \mathcal{A}=\left\{A_{Q_{K}}\right\}
$$

where $A_{Q_{K}}$ defines a state space representation for $Q_{K}\left(\frac{d}{d t}\right) w=0, Q_{K} \in \mathcal{Q}_{\mathcal{K}}$ obtained from the same statemap. In the last decade many conditions have been derived that guarantee the existence of a common Lyapunov function for a set of LTI systems $[5,16]$. The majority of such conditions consider the existence of a common quadratic Lyapunov function (CQLF) $V(x)=x^{T} L x$ with $L=L^{T}>0$ such that the linear matrix inequalities (LMIs) $A_{Q}^{T} L+L A_{Q}<0 \forall Q \in \mathcal{Q}_{\mathcal{K}}$. Convex optimization tools can be used to check the feasibility of such a set of LMIs. However, this numerical approach fails to give much insight into the stability or instability mechanisms of the system and does not supply any guidelines for designing stable switched systems. Further, as we shall show in this paper, there are problems of practical importance where this LMI test fails to establish both the existence, or inexistence of a CQLF, and is therefore useless in such situations.

A number of analytic conditions for the existence of a CQLF for several sub-classes of switched systems have been derived in the recent past. [15, 14]. All these results suffer from the shortcoming that the structural assumptions made on the system matrices only hold for a small class of systems. Also, these assumptions are seldom robust against numerical perturbations.

The contributions of this paper are twofold: first, and foremost, we provide an algorithmic method to construct a family of differential algebraic systems that share a quadratic Lyapunov function. Results here build on our earlier results [9] where we obtained a characterization for a cone of matrices that have a CQLF. This parametrization was obtained as a sufficient condition in terms of certain constant matrices. We extend these results here and enlarge the parametrized set by also allowing a certain type of polynomial matrices. The second contribution of our paper is an investigation of several important system theoretic problems that can be formulated as stability problems for switched systems. Specifically we consider design of controllers that ensure stability against arbitrary sensor/actuator failure, and a design procedure for switched controllers. Our results are not only theoretically powerful, but also suitable for numerical computation. We show that the characterization can be used in practice by solving an associated LMI.

\section{MOTIVATING EXAMPLE - NITRIFYING TRICKLING FILTER}

The most generally applicable methods to analyze the quadratic stability of switched systems have been derived from a computational point of view e.g. [3]. Many stability criteria are formulated as LMIs that can be solved efficiently, thanks to recent developments in convex optimization. However, there are examples of practical concern where purely numerical approaches do not work, and hence an analytical investigation becomes imperative. The following example demonstrates that LMI based conditions for checking existence of CQLFs can fail even in apparently simple situations.

We consider a model for a nitrifying trickling filter (NTF) proposed by Wik and Breitholtz [21]. This filter oxidizes ammonium in wastewater into nitrate. The transfer function from inlet to outlet nitrate concentrations is given by

$$
G_{1}(s)=\left(\frac{0.435}{1+1.0796 s}+\frac{0.548}{1+0.3124 s}+0.016\right)^{10}
$$

Feedback control schemes are used in order to achieve a desired nitrate concentration at the outlet. To keep things simple, we consider a unity feedback and define

$$
G_{2}(s)=\frac{G_{1}}{1+G_{1}}
$$

and investigate whether the autonomous dynamics associated with $G_{1}$ and $G_{2}$ remain stable under arbitrary switching. This is a practically relevant scenario since it investigates whether the control loop remains stable under intermittant or permanent sensor and/or actuator failure. Thus, the problem of stability analysis under sensor failure is reduced to one of stability analysis of switched autonomous dynamical systems.

Let $\dot{x}=A_{1} x$ and $\dot{x}=A_{2} x$ be state space descriptions for the autonomous dynamics of $G_{1}$ and $G_{2}$. Both $A_{1}$ and $A_{2}$ are Hurwitz. We search for a CQLF for the two systems. It is known from convex optimization theory that exactly one of the following must hold [1] 
(1) There exists $L=L^{T}>0$ such that $A_{i}^{T} L+L A_{i}<0$, $i=1,2$

(2) There exist $R_{i}=R_{i}^{T}>0$ such that $\sum_{i=1}^{2} A_{i} R_{i}+$ $R_{i} A_{i}^{T}>0$.

That is, if (1) holds, we know there exists a CQLF, and if (2) holds we know there does not exist a CQLF. However, it is seen that the commonly available LMI solver [8] is unable to solve either of the two LMIs, and therefore the LMI condition fails to answer the question whether there exists a CQLF for the two system, or there doesn't.

Thus there is a case for analytical results for answering the question of existence or inexistence of CQLFs. In the remaining sections of the paper, we develop analytical results that establish sufficient conditions for a finite number of autonomous linear systems to have an asymptotically stable equilibrium under arbitrary switching. We then use our results to re-address the example considered above and show how our method yields insights into analysis of switched systems.

\section{BEHAVIORAL THEORY}

In recent years, the behavioral theory of dynamical systems has emerged as an alternative to input-output (transfer function or state-space) based system analysis. Details of the behavioral approach can be found in [11].

\subsection{Linear Differential Systems}

A behavior is, broadly speaking, a collection of trajectories in a pre-defined function space (e.g. the space of locally integrable functions), characterized by certain laws. If these laws are linear and time-invariant, the corresponding behavior is called Linear, Time-invariant (LTI). A linear differential behavior is one in which the behavior can be characterized as the solution set of a family of linear Ordinary Differential Equations (ODEs). The image representation plays an important role in the behavioral framework: an LTI system $\Sigma$ with external variables ("inputs" and "outputs") $(u, y)$ is controllable if and only if it can be represented as the image of a linear differential operator acting on free variables in an appropriate space:

$$
\left[\begin{array}{l}
u \\
y
\end{array}\right]=\left[\begin{array}{l}
Q\left(\frac{d}{d t}\right) \\
P\left(\frac{d}{d t}\right)
\end{array}\right] \ell
$$

where $Q \in \mathbb{R}^{\mathrm{q} \times \mathrm{q}}[D]$, and $P \in \mathbb{R}^{\mathrm{p} \times \mathrm{q}}[D]$ are polynomial differential operators. The indeterminate " $D$ " denotes symbolic differentiation. The free variable $\ell$, also called a latent variable, is assumed to lie in $\mathcal{L}_{1}^{\text {loc }}\left(\mathbb{R}, \mathbb{R}^{\mathrm{q}}\right)$, the space of locally integrable functions from $\mathbb{R}$ to $\mathbb{R}^{q}$. Since $\ell$ is free, one can assume $Q$ and $P$ to be right coprime without loss of generality. The partition of the system variables as $(u, y)$ in Equation (3) is called an input-output partition, with inputs $u$ and outputs $y$ if $Q$ is square and nonsingular, and the rational function $P Q^{-1}$ is proper.

A system can be given by several representations, in terms of inputs, outputs and internal variables. Internal variables that satisfy an "axiom of state" [12] are called "states", and system representations in terms of these variables are called state representations. A representation is a state representation if and only if it is first-order in terms of states, and zeroth order in terms of inputs and outputs. Given a controllable system, having behavior $\mathcal{B}$ as defined in Equation (3), one can construct a polynomial differential operator $X\left(\frac{d}{d t}\right)$ such that variables $x$ defined as

$$
x=X\left(\frac{d}{d t}\right)\left[\begin{array}{l}
u \\
y
\end{array}\right],(u, y) \in \mathcal{B}
$$

are state variables. The operator $X\left(\frac{d}{d t}\right)$ is called a state map. With $(u, y)$ an input-output partition of a behavior defined by (3), $Q \in \mathbb{R}^{\mathrm{q} \times \mathrm{q}}[D]$, the span of rows of the polynomial matrix $X(D)$ (over $\mathbb{R}$ ) is precisely the span of rows $r_{i}$ (over $\mathbb{R}$ ) such that $r_{i} Q^{-1}$ is strictly proper. In particular if $Q(D)=\sum_{i=0}^{d} \mathrm{Q}_{i} D^{i}$ is regular, $X\left(\frac{d}{d t}\right)$ can be defined by the polynomial differential operator

$$
X\left(\frac{d}{d t}\right)=\left[\begin{array}{llll}
I & I \frac{d}{d t} & \cdots & I \frac{d^{d-1}}{d t^{d-1}}
\end{array}\right]^{T}
$$

It is easy to see that the above state map transforms the system with image representation (3) into a "blockcompanion" form, and further this state representation is minimal in terms of the number of states among all possible state representations.

\subsection{Dissipative Systems}

We first introduce the concept of Quadratic Differential Forms (QDFs) that are central to the discussion in this section. In Lyapunov theory, optimal control etc., we often encounter quadratic functionals of variables and their derivatives (e.g. Lyapunov function, the cost functional, the Lagrangian etc.). In [20] a two variable polynomial matrix was used to represent such quadratic functionals. Consider $w \in \mathfrak{L}_{1}^{\text {loc }}\left(\mathbb{R}, \mathbb{R}^{\mathrm{w}}\right)$. Consider $\Phi \in \mathbb{R}^{\mathrm{w} \times \mathrm{w}}[\zeta, \eta]$ given by

$$
\Phi(\zeta, \eta)=\sum_{k, l} \Phi_{k, l} \zeta^{k} \eta^{l}
$$

where $\Phi_{k, l} \in \mathbb{R}^{\mathrm{w} \times \mathrm{w}}$ and the sum ranges over non-negative integers $k, l$. This sum is assumed finite (i.e. only a finite number of $\Phi_{k l}$ are nonzero). Such a $\Phi$ induces a quadratic differential form (QDF) defined by

$$
\left(Q_{\Phi}(w)\right)(t)=\sum_{k, l}\left(\frac{d^{k} w(t)}{d t^{k}}\right)^{T} \Phi_{k l}\left(\frac{d^{l} w(t)}{d t^{l}}\right) .
$$

where the derivative is in the sense of locally integrable functions. Due to the quadratic nature of $Q_{\Phi}$, differentiability requirements may impose additional structural restrictions on $\Phi$ in order to ensure that $Q_{\Phi}(w)$ is also locally integrable.

We review basic properties of dissipative systems in this section. The abstract theory of dissipative systems was introduced by Willems, who in 1972 wrote two seminal papers on the subject [19]. The ideas in these papers have been singularly successful in tieing together concepts from network theory, mechanical systems, thermodynamics, and feedback control theory. The dissipation hypothesis which distinguishes dissipative systems from general dynamical systems results in a fundamental constraint on their dynamical behavior. Consider the system (3) having behavior $\mathcal{B}$, with $Q \in \mathbb{R}^{\mathrm{q} \times \mathrm{q}}[D], P \in \mathbb{R}^{\mathrm{p} \times \mathrm{q}}[D]$. Define $\mathrm{m}:=\mathrm{q}+\mathrm{p}$. Consider $\Phi \in \mathbb{R}^{\mathrm{m} \times \mathrm{m}}[\zeta, \eta] . \mathcal{B}$ is called $\Phi$-dissipative if 


$$
\int_{-\infty}^{\infty} Q_{\Phi}(u, y) d t \geq 0 \quad \forall(u, y) \in \mathcal{B} \cap \mathcal{D}\left(\mathbb{R}, \mathbb{R}^{\mathrm{m}}\right) .
$$

In the above inequality $\mathcal{D}\left(\mathbb{R}, \mathbb{R}^{\mathrm{m}}\right)$ denotes the space of compactly supported locally integrable functions from $\mathbb{R}$ to $\mathbb{R}^{\mathrm{m}}$. The system (3) is $\Phi$-dissipative if and only if

$$
\left[Q^{T}(-i \omega) P^{T}(-i \omega)\right] \Phi(-i \omega, i \omega)\left[\begin{array}{l}
Q(i \omega) \\
P(i \omega)
\end{array}\right] \geq 0, \forall \omega \in \mathbb{R}
$$

The function $Q_{\Phi}$ is called a "supply function" and is a measure of the generalized power supplied. Also associated with a dissipative system is a function $Q_{\Psi}$, called a "storage function", that satisfies the so called "dissipation inequality":

$$
\frac{d}{d t} Q_{\Psi}(u, y) \leq Q_{\Phi}(u, y)
$$

Note that there may exist nonzero trajectories along which $\frac{d}{d t} Q_{\Psi}(u, y)$ exactly equals the supply $Q_{\Phi}$. This is undesirable in some problems, especially in stability analysis. Therefore, we define a set of "strictly" dissipative systems:

Definition 1. Let $G=P Q^{-1}$ with $P \in \mathbb{R}^{\mathrm{p} \times \mathrm{q}}[D], Q \in$ $\mathbb{R}^{\mathrm{q} \times \mathrm{q}}[D]$ regular. With $\mathrm{m}=\mathrm{p}+\mathrm{q}$, consider $\Phi \in \mathbb{R}^{\mathrm{m} \times \mathrm{m}}[\zeta, \eta]$. The behavior $\mathcal{B}$ defined by the image representation (3) is called strictly $\Phi$-dissipative if $\exists \epsilon>0$ such that $\mathcal{B}$ is $\left(\Phi-\epsilon I_{\mathrm{m}}\right)$-dissipative.

Note that along every nonzero trajectory in a strictly $\Phi$-dissipative system, there exists $Q_{\Psi}$ such that $\frac{d}{d t} Q_{\Psi}$ is strictly less than $Q_{\Phi}$.

In the sequel, we consider supply functions $Q_{\Phi}$, that have the following structure:

$$
\Phi=\left[\begin{array}{cc}
\Theta_{11} & \Theta_{12}^{T}(\eta) \\
\Theta_{12}(\zeta) & 0_{\mathrm{p}}
\end{array}\right]
$$

$\Theta_{11} \in \mathbb{R}^{\mathrm{q} \times \mathrm{q}}>0_{\mathrm{q}}$, and $\Theta_{12}(D) \in \mathbb{R}^{\mathrm{p} \times \mathrm{q}}[D]$. The choice of this structure is motivated by the stability problem considered in this paper. Note in particular that $Q_{\Phi}(0, y)=0$ for all $y \in \mathbb{R}^{\mathrm{p}}$. In order to ensure that $Q_{\Phi}$ is well defined along a behavior, we only consider those behaviors associated with the proper rational function $P Q^{-1}$ such that $\operatorname{deg} \Theta_{12} \leq \operatorname{deg} Q-\operatorname{deg} P$. This is a standing assumption we make throughout the paper, unless otherwise stated.

The following theorem investigates under what conditions do there exist positive definite storage functions for strictly $\Phi$-dissipative systems.

Theorem 2. Let $\mathcal{B}$ defined by (3) with $P Q^{-1}$ proper be strictly $\Phi$-dissipative, where $\Phi \in \mathbb{R}^{\mathrm{m} \times \mathrm{m}}[\zeta, \eta]$ as in (9). If all roots of $\operatorname{det} Q=0$ lie in the open left half complex plane, every storage function of $\mathcal{B}$ with respect to $Q_{\Phi}$ is a positive definite state function.

\section{SWITCHED AUTONOMOUS SYSTEMS}

We now address the problem of constructing switched linear systems (2) whose component systems have a CQLF. We characterize the component systems in terms of an image representation of an associated strictly dissipative system. This approach, as we shall show, has many advantages. We shall present some preliminary results in order to obtain the characterization mentioned above.

Lemma 3. Let $\Phi \in \mathbb{R}^{\mathrm{m} \times \mathrm{m}}[\zeta, \eta]$ be given as in (9) and consider a strictly $\Phi$-dissipative behavior $\mathcal{B}$ defined by (3),
$Q$ regular and Hurwitz, with $\operatorname{deg} \Theta_{12} \leq \operatorname{deg} Q-\operatorname{deg} P$. Let $\mathrm{P}_{h}$ be the highest degree coefficients of $P$ and $\Theta_{12 h}$ be the coefficient of the term $\zeta^{\operatorname{deg} Q-\operatorname{deg} P}$ in $\Theta_{12}(\zeta)$ (possibly zero). Then, $\Theta_{11}+\Theta_{12 h}^{T} \mathrm{P}_{h}+\mathrm{P}_{h}^{T} \Theta_{12 h}>0$.

Given a supply function $Q_{\Phi}$, the following Lemma provides for the construction of another associated supply function that will be used in the sequel:

Lemma 4. Let $\Phi_{1}$ be of the form (9) and $S(D)=$ $\left[\begin{array}{cc}I_{\mathrm{q}} & K(D) \\ 0 & I_{\mathrm{p}}\end{array}\right]$ with $K(D) \in \mathbb{R}^{\mathrm{q} \times \mathrm{p}}[D]$ such that there exists a polynomial matrix $R(D) \in \mathbb{R}^{\mathrm{p} \times} \bullet[D]$ satisfying

$$
\left[K^{T}(\zeta) I_{\mathrm{p}}\right]\left[\begin{array}{cc}
-\Theta_{11} & \Theta_{12}^{T}(\eta) \\
\Theta_{12}(\zeta) & 0_{\mathrm{p}}
\end{array}\right]\left[\begin{array}{c}
K(\eta) \\
I_{\mathrm{p}}
\end{array}\right]=R^{T}(\zeta) R(\eta) .
$$

Define $\Phi_{2}=S^{-T}(\zeta) \Phi_{1} S^{-1}(\eta)$. Then $Q_{\Phi_{2}}(0, y) \leq 0$ for all $y \in \mathbb{R}^{\mathrm{p}}$.

The following lemma gives bounds on the degree of $K(D)$ in lemma 4

Lemma 5. Consider $K(D)$ as defined in Lemma 4. Then, $\operatorname{deg} K \leq \operatorname{deg} \Theta_{12}$.

Consider a supply function $Q_{\Phi_{1}}$ and a $\Phi_{1}$-dissipative behavior $\mathcal{B}_{1}$. Using the construction of the supply function $Q_{\Phi_{2}}$ in Lemma 4, we construct a $\Phi_{2}$-dissipative behavior having the same storage functions as $\mathcal{B}_{1}$ with respect to $Q_{\Phi_{1}}$ :

Theorem 6 . Let $\Phi_{1}$ and $\Phi_{2}$ be such that they satisfy the conditions in Lemma 4. Let $\mathcal{B}_{1}=\left[\begin{array}{c}Q_{1}\left(\frac{d}{d t}\right) \\ P_{1}\left(\frac{d}{d t}\right)\end{array}\right] \ell$, with $Q_{1}$ regular and Hurwitz, $P_{1} Q_{1}^{-1}$ proper, be strictly $\Phi_{1^{-}}$ dissipative. Define $\mathcal{B}_{2}=\left[\begin{array}{c}Q_{2}\left(\frac{d}{d t}\right) \\ P_{2}\left(\frac{d}{d t}\right)\end{array}\right] \ell$ where
$\left[\begin{array}{l}Q_{2}(D) \\ P_{2}(D)\end{array}\right]=S \cdot\left[\begin{array}{l}Q_{1}(D) \\ P_{1}(D)\end{array}\right]$

Then, $\mathcal{B}_{2}$ has the following properties:

(1) $Q_{2}$ is regular and $\operatorname{deg} Q_{2}=\operatorname{deg} Q_{1}$.

(2) $Q_{2}, P_{2}$ are right coprime.

(3) $\mathcal{B}_{2}$ is strictly $\Phi_{2}$-dissipative.

(4) Every storage function (on states) of $\mathcal{B}_{2}$ with respect to $Q_{\Phi_{2}}$ is also a storage function (on states) of $\mathcal{B}_{1}$ with respect to $Q_{\Phi_{1}}$.

Having established the existence of a common storage function we now present the following central stability result of this paper:

Theorem \%. Consider a strictly $\Phi$-dissipative behavior $\mathcal{B}$ defined by (3) with $Q$ regular and Hurwitz. Define

where $K$ satisfies

$$
Q_{K}(D)=\left[\begin{array}{ll}
I_{\mathrm{q}} & K(D)
\end{array}\right]\left[\begin{array}{l}
Q(D) \\
P(D)
\end{array}\right]
$$

$$
\left[K^{T}(\zeta) I_{\mathrm{p}}\right]\left[\begin{array}{cc}
-\Theta_{11} & \Theta_{12}^{T}(\eta) \\
\Theta_{12}(\zeta) & 0_{\mathrm{p}}
\end{array}\right]\left[\begin{array}{c}
K(\eta) \\
I_{p}
\end{array}\right]=R^{T}(\zeta) R(\eta) .
$$

for some $R(D) \in \mathbb{R}^{\mathrm{p} \times} \bullet[D]$. Define $\Sigma_{\mathcal{Q}}:=\mathcal{Q} \frac{d}{d t}(t) w=0$ with $\mathcal{Q}(D) \in\left\{Q_{K}\right\}$. Then, the equilibrium state 0 in $\Sigma_{\mathcal{Q}_{\mathcal{K}}}$ is uniformly exponentially stable under arbitrary switching. 
Remark 8. Theorem 7 not only gives a characterization of stabilizing feedback controllers but also suggests a computationally feasible scheme to compute these controllers. Consider $\Phi=\left[\begin{array}{cc}\Theta_{11} & \Theta_{12}^{T} \\ \Theta_{12} & 0_{\mathrm{p}}\end{array}\right] \in \mathbb{R}^{\mathrm{q}+\mathrm{p} \times \mathrm{q}+\mathrm{p}}$. The inequality

$$
\left[\begin{array}{ll}
K^{T} & I_{\mathrm{p}}
\end{array}\right]\left[\begin{array}{cc}
-\Theta_{11} & \Theta_{12}^{T} \\
\Theta_{12} & 0_{\mathrm{p}}
\end{array}\right]\left[\begin{array}{c}
K \\
I_{\mathrm{p}}
\end{array}\right]>0
$$

is quadratic in $K$. However, using the Schur complement we can re-write (10) as a linear inequality in $K$. Indeed, (10) holds if and only if

$$
\left[\begin{array}{cc}
\Theta_{11}^{-1} & K \\
K^{T} & K^{T} \Theta_{12}^{T}+\Theta_{12} K
\end{array}\right]>0
$$

Inequality (11) can be solved as an LMI to determine a feasible $K$. Further constraints may be imposed on $K$ as required.

\section{EXAMPLE - NITRIFYING TRICKLING FILTER REVISITED}

We now reconsider the example in Section 4 and use results obtained so far to address the problem of designing output feedback controllers that are robust against arbitrary switching, caused by sensor or actuator failure. First, we identify a supply function for the system defined by $G_{1}$. In the SISO case, the Nyquist Plot of $G_{1}$ can be used to obtain a supply function

$$
\Phi=\left[\begin{array}{ll}
1 & 1 \\
1 & 0
\end{array}\right]
$$

From Theorem 7 we observe that with

$$
\left[\begin{array}{ll}
k & 1
\end{array}\right]\left[\begin{array}{cc}
-1 & 1 \\
1 & 0
\end{array}\right]\left[\begin{array}{l}
k \\
1
\end{array}\right] \geq 0
$$

the autonomous dynamics associated with $G_{1}$ and $\frac{G_{1}}{1+k G_{1}}$ remain stable under arbitrary switching. Thus, $k \in[0,2]$ stabilizes $G_{1}$ and also ensures that the closed loop remains stable under sensor/actuator failure. Hence, the unity feedback scheme considered in Section 4 satisfies the property that the open and closed loop systems remain stable under arbitrary switching.

\section{FURTHER APPLICATIONS}

In this section we list further applications of Theorem 7 . These applications serve to demonstrate the flexibility of our approach.

\subsection{Sensor/Actuator Failure}

Evaluation of controller robustness against sensor/actuator failure is of immense interest. Instability could result because of not only high feedforward gain, but also because of switching among nominally stable systems, corresponding to each failure scenario. Thus the problem of analysing stability of a closed loop system under sensor/actuator failure can be formulated as one of analysing stability of a switched system with several components.

As already demonstrated in Sections 4 and 7, an immediate application of Theorem 7 is that it guarantees stability under loop disruptions caused by sensor failure. Note that Theorem 7 also holds for $K=0$. Thus, the open loop system and the closed loop system remain stable under arbitrary switching provided $K$ satisfies conditions in Theorem 7 .

Stronger results may be obtained when special structure is imposed on the feedback controllers. Let the open loop plant (3) with $P, Q \in \mathbb{R}^{\mathrm{q} \times \mathrm{q}}[D]$ be (strictly) dissipative with respect to

$$
\Phi=\left[\begin{array}{ll}
\Gamma & I_{\mathrm{q}} \\
I_{\mathrm{q}} & 0_{\mathrm{q}}
\end{array}\right]
$$

with $\Gamma=\operatorname{diag}\left(\gamma_{1}, \ldots, \gamma_{\mathrm{q}}\right)$. It can be shown for every $P Q^{-1}$ proper, there exists a $\Gamma$ such that $\mathcal{B}$ is $\Phi$-dissipative.

Application of Theorem 7 results in the following condition that a feedbacks $K$ must satisfy in order that $\operatorname{Ker} Q\left(\frac{d}{d t}\right)$ and $\operatorname{Ker}\left(Q\left(\frac{d}{d t}\right)+K P\left(\frac{d}{d t}\right)\right)$ remain stable under arbitrary switching:

$$
-K^{T} \Gamma K+K+K^{T} \geq 0
$$

Consider a nominal $K=\operatorname{diag}\left[\alpha_{1}, \ldots, \alpha_{\mathrm{q}}\right]$ that satisfies (13), i.e. $\alpha_{i} \in\left[0,2 / \gamma_{i}\right], i=1, \ldots, \mathrm{q}$. Under these conditions, the closed loop remains stable under arbitrary combinations of sensor or actuator failure since the condition (13) still holds true when some of the $\alpha_{i}$ s are replaced by 0 . Thus we have obtained bounds on the feedback gains $\alpha_{i}$ which ensures that the autonomous dynamics remains stable under arbitrary sensor or actuator failure.

In the special case when some $\gamma_{i}=0$, there is no upper bound on $\alpha_{i}$ s, i.e. arbitrary negative feedback between the $i$ th output and input still ensures that the switched system remains asymptotically stable under actuator or sensor failures.

Analysing the problem of stability under sensor/actuator failure by solving a family of LMIs for a CQLF is quite inefficient. Clearly, with $K=\operatorname{diag}\left(\alpha_{1}, \ldots, \alpha_{\mathrm{q}}\right)$ there exist $2^{\mathrm{q}}$ failure scenarios, and hence also $2^{\mathrm{q}}$ LMIs which need be solved simultaneously. This is a computationally difficult problem for large q.

\subsection{Switched Controller Design}

The problem of controller design is as follows: one desires controllers $K_{i}, i=1, \ldots N$ that satisfy certain specifications and ensure stability under arbitrary switching. In order to obtain such controllers, we first obtain a supply function $Q_{\Phi}$ such that the open loop plant defined by $G$ is strictly $\Phi$-dissipative. It is shown in (11) that $K_{i} \mathrm{~s}$ may be determined by solving an LMIs. The interesting aspect of this condition is that structural conditions that are difficult to handle analytically may now be imposed on $K$. For example, one can search for a diagonal $K$ which satisfies the conditions in Theorem 7. In the light of Section 8.1 , one may also want to design controllers that render the open loop plant dissipative with respect to "special" supply functions, for instance (12). Such designs ensure robustness against arbitrary combinations of sensor and actuator failure. We demonstrate this application in the simple example below. In [17], Page 93, a simplified model for a distillation column is proposed:

$$
G(s)=\frac{1}{75 s+1}\left[\begin{array}{cc}
87.8 & -86.4 \\
108.2 & -109.6
\end{array}\right]
$$

We design a output feedback controller in order to have a certain desired pole location. We would in addition like 
this controller to ensure stability against actuator failure. With $Q=\operatorname{diag}(1+75 s, 1+75 s)$ and $P=\left[\begin{array}{cc}87.8 & -86.4 \\ 108.2 & -109.6\end{array}\right]$, it can be seen that the behavior $\mathcal{B}$ associated with $y=G u$ is dissipative with respect to $Q_{\Phi}$ with

$$
\Phi(\zeta, \eta)=\left[\begin{array}{cccc}
5 & 0 & 0 & 1 \\
0 & 5 & -1 & -\eta \\
0 & -1 & 0 & 0 \\
1 & -\zeta & 0 & 0
\end{array}\right]
$$

We note that $K(D)=\left[\begin{array}{cc}0 & 0.4 \\ -0.4 & -0.4 D\end{array}\right]$ meets the conditions in Theorem 7 with $R=0_{2}$. Indeed it can be verified that with an output feedback defined by $K\left(\frac{d}{d t}\right)$, the closed loop poles move from $\{-0.0133,-0.0133\}$ to $\{-0.005,-0.67\}$.

Note that the output feedback represented by $K_{0}=0_{2}$, $K_{1}=\left[\begin{array}{cc}0 & 0 \\ -0.4 & -0.4 D\end{array}\right]$ and $K_{2}=\left[\begin{array}{cc}0 & 0.4 \\ 0 & 0\end{array}\right]$ represent possible actuator failure scenarios. Under these conditions, $K_{i}(D), i=0 \ldots, 2$ still satisfy the condition in Theorem 7 . Therefore the autonomous dynamics $\operatorname{Ker} Q\left(\frac{d}{d t}\right), \operatorname{Ker}(Q+$ $\left.K_{1} P\right)\left(\frac{d}{d t}\right), \operatorname{Ker}\left(Q+K_{2} P\right)\left(\frac{d}{d t}\right)$ and $\operatorname{Ker}(Q+K P)\left(\frac{d}{d t}\right)$ remain stable under arbitrary switching. LMI tests in fact show that there also exists a CQLF, as already predicted in Theorem 7:

$$
L=\left[\begin{array}{cc}
0.4657 & -0.4543 \\
-0.4543 & 0.5342
\end{array}\right]
$$

is a $\mathrm{CQLF}$ for $\operatorname{Ker} Q\left(\frac{d}{d t}\right), \operatorname{Ker}\left(Q+K_{1} P\right)\left(\frac{d}{d t}\right), \operatorname{Ker}(Q+$ $\left.K_{2} P\right)\left(\frac{d}{d t}\right)$ and $\operatorname{Ker}(Q+K P)\left(\frac{d}{d t}\right)$. Thus, the closed loop system with output feedback defined by $K\left(\frac{d}{d t}\right)$ not only ensures closed loop stability, but also ensures robustness against arbitrary actuator failure.

\section{CONCLUSION}

We propose a parametrization for a set of autonomous differential algebraic systems that have a Common Quadratic Lyapunov Function (CQLF). Switched systems, with components from this set remain stable under arbitrary switching. The parametrizations can be efficiently computed by solving an LMI. Our results can be used even where commonly available numerical approaches apparently fail, as demonstrated by the Trickling Nitrifying Filter example. We also address related problems of stability under arbitrary sensor/actuator failure and controller design which we use for a simplified distillation column control.

\section{REFERENCES}

[1] V. Balakrishnan and L. Vandenberghe. Semidefinite programming duality and linear time-invariant systems IEEE Trans. Aut. Control 48(1):30-41, 2003.

[2] R. DeCarlo, M. Branicky, S. Pettersson, and B. Lennartson. Perspectives and results on the stability and stabilisability of hybrid systems. Proceedings of the IEEE, 88(7):1069-1082, 2000.

[3] M. K.-J. Johanson. Piecewise Linear Control Systems. Springer-Verlag, 2002.

[4] D. Liberzon. Switching in Systems and Control. Birkhäuser, 2003.
[5] D. Liberzon and A. S. Morse. Basic problems in stability and design of switched systems. IEEE Control Systems Magazine, 19(5):59-70, 1999.

[6] A. P. Molchanov, and E. S. Pyatnitskii. Criteria of asymptotic stability of differential and difference inclusions encountered in control theory. Systems and Control Letters, 1:59-64, 1989.

[7] K. S. Narendra, and J. Balakrishnan, A common Lyapunov function for stable LTI systems with commuting $\mathcal{A}$-matrices. IEEE Trans. Aut. Control, 39:24692471, 1994.

[8] R. Nikoukhah, F. Delebecquey, L. El Ghaouiz. LMITOOL: a Package for LMI Optimization in Scilab-Users Guide. www.scilab.org/doc/lmidoc/lmi.pdf, August 2007.

[9] I. Pendharkar, K. Wulff, and J. Raisch. A sufficient condition for quadratic stability of switched linear systems with multiple component systems. IEEE Conference on Decision and Control, New Orleans, USA, 2007.

[10] I. Pendharkar, K. Wulff, and J. Raisch. On the stability of switched differential algebraic systems: conditions and applications. Technical Report, Forschungsberichte der Fakultät IV, Technische Universität Berlin, 2007/17, 2007.

[11] J. W. Polderman, J. C. Willems, Introduction to Mathematical Systems Theory: A Behavioral Approach. Springer-Verlag, 1997.

[12] P. Rapisarda and J.C. Willems. State maps for linear systems. SIAM Journal of Control and Optimization, 35:1053-1091, 1997.

[13] A.J. van der Schaft, H. Schumacher. An Introduction to Hybrid Dynamical Systems. LNCIS 251, SpringerVerlag, 2000.

[14] R.N. Shorten, and K.S. Narendra. Necessary and sufficient conditions for the existence of a common quadratic Lyapunov function for M-stable linear second order systems. Proc American Control Conference, 2000.

[15] R. N. Shorten and F. Ó Cairbre. A new methodology for the stability analysis of pairwise triangular and related switching systems. Institute of Mathematics and its Applications: Journal of Applied Mathematics, 67:441-457, 2002.

[16] R. Shorten, F. Wirth, O. Mason, K. Wulff, and C. King. Stability criteria for switched and hybrid systems. SIAM review, 49(4), pp. 545-592, 2007.

[17] S. Skogestad, I. Postlethwaite. Multivariable Feedback Control. John Wiley, 1996.

[18] H.L. Trentelman and J.C. Willems. Every storage function is a state function. Systems and Control letters, 32:249-259, 1997.

[19] J.C. Willems. Dissipative dynamical systems, Parts 1 \& 2. Archives for Rational Mechanics and Analysis, 45, pp 321-351, pp 352-393, 1972.

[20] J.C. Willems and H.L. Trentelman. On Quadratic Differential Forms. SIAM Journal of Control and Optimization, 36:1703-1749, 1998.

[21] T. Wik, C. Breitholz. Rational Transfer Function Models for Biofilm Reactors. Process Systems Engineering 44(12):2647-2657, 1998. 